\title{
Sobreprecio y acceso a los medicamentos: el caso de los medicamentos esenciales en México
}

\author{
O verpricing and affordability of drugs: \\ the case of essential drugs in M exico
}

Raúl E. Molina-Salazar 1 José F. Rivas-Vilchis 2

\footnotetext{
1 Departamento de Economía, Universidad Autónoma Metropolitana - Iztapalapa. Michoacán y Purísima s/no, Iztapalapa, DF, México 09340.

2 Departamento de

Atención a la Salud,

Universidad Autónoma Metropolitana - Xochimilco Calz. del Hueso 1100 , Coyoacan, DF, México 04960. jfrivas@cueyatl.uam.mx
}

Abstract Accessibility and availability of drugs has been a matter of great concern for health services all over the world, especially for less developed countries. TheWorld Health Organization has devoted considerable time to this matter. Although the essential drug policy has been well-accepted by health agencies and NGOs, the pharmaceutical industry has not proven willing to produce essential drugs at affordable prices. The purpose of this study is to examine pricelevels of essential drugs in Mexico. The evaluation was performed through a comparison of international and national prices for leading drugs in the respective therapeutic categories and included in the WHO model list of essential drugs. The study shows clearly that prices of essential brand-namedrugs in Mexico are very high. Per capita consumption has remained stable despite a sharp decrease in the Mexican GDP since 1995. The article discusses the reasons for this and proposes measures to deal with the problem.

Key words Essential Drugs; Drug Prices; Health Policy

Resumen Para la Organización Mundial dela Salud (OMS) la accesibilidad y la disponibilidad de los medicamentos es un tema central al cual leha dedicado una gran atención. Si bien la política de medicamentos esenciales ha teni do una acogida favorable por parte de las organizaciones sanitarias, la industria farmacéutica, especialmente sus filiales asentadas en los países menos desarrol lados, no ha brindado una respuesta adecuada a los planteami entos de la OMS de proporcionar medicamentos esenciales a precios accesibles. El objetivo de este trabajo es estudiar el precio de los medicamentos esenciales de marca en México en relación al precio delos medi camentos esenciales genéri cos disponi bles en el mercado internacional. Se estudió una muestra de especiali dades farmacéuticas líderes de ventas en su categoría terapéutica y se compararon los precios, por unidades farmacéuticas, de los medicamentos de marca y los medicamentos genéricos a precio internacional. Los resultados muestran que el nivel de precios de los medicamentos esenciales en Méxi co es muy el evado. La venta per cápita no se ha reducido, a pesar dela caída del producto interno bruto que se produjo en México a partir del año de 1995. Se discuten las razones de este sobreprecio y se proponen al gunas medidas para sol uci onar este problema.

Palabras clave Medicamentos Esenciales; Precios de Medicamentos; Políticas de Salud 


\section{Introducción}

El Director General de la Organización Mundial de la Salud (OMS) envió, en 1975, a la 28a Asamblea Mundial de Salud un reporte, en el cual se revisaban los principales problemas que enfrentaban los países en desarrollo respecto a los medicamentos y se delineaban políticas nuevas respecto a la utilización de medicamentos (WHO, 1975).

La OMS crea la primera lista modelo de medicamentos esenciales (WHO, 1977), la cual sirvió de base a diferentes países para instituir formularios nacionales. Esta lista ha sido actualizada en diversas ocasiones hasta su última versión (WHO, 1997). En estos reportes se señala que la selección de medicamentos esenciales depende de las necesidades sanitarias, la estructura y el desarrollo de los servicios de salud de cada país. De esta manera, las listas de medicamentos esenciales deben ser elaboradas localmente y ser actualizadas periódicamente, con la asesoría de expertos en salud pública, medicina, farmacología, farmacia y administración de medicamentos. Por otra parte, en 1981, la OMS adoptó la estrategia global de "Salud para todos en el año 2000", y uno de los aspectos centrales de esta estrategia es garantizar el abastecimiento adecuado de los productos farmacéuticos esenciales a un costo accesible, en especial a los países menos desarrollados (PMD) (WHO, 1981).

La producción y el consumo de fármacos de medicamentos en el mundo se encuentran marcadamente concentrados en los países desarrollados (PD). Los PD de economía de mercado y ex-socialistas generan el $89 \%$ de la producción de medicamentos. Estudios que comparan el gasto en medicamentos en los PD con el de los países menos desarrollados (PMD) muestran que a los primeros corresponde más del $70 \%$ del consumo mundial de los productos farmacéuticos (WHO/DAP, 1997).

Los países con mayores necesidades sanitarias que a la vez tienen menores ingresos destinan frecuentemente una proporción insuficiente a gastos en salud, y esto generalmente es acompañado por el uso irracional de los medicamentos (Chetley, 1989).

I gualmente, los PMD dedican, desde el desarrollo masivo de la industria farmacéutica, una proporción excesiva de sus presupuestos de salud a la adquisición de medicamentos, y el gasto en productos farmacéuticos representa comúnmente un al to porcentaje ( 20 a 45\%) del Producto Interno Bruto (PIB) destinado a la conservación de la salud (Patel, 1983; Murray \& Lopez, 1994). En especial, los países de Améri- ca Latina aplican aproximadamente el $25 \%$ de su presupuesto en salud a la adquisición de productos farmacéuticos, mientras que los PD de América del Norte, de Europa Occidental y Europa Oriental gastan, aproximadamente, $15 \%$ de los recursos destinados a salud en la obtención de medicamentos (Burstall, 1990).

A pesar del alto porcentaje del PIB que destinan Ios PMD en la adquisición de medicamentos se dispone generalmente en estos países con menos de 10 dólares per cápita por año para la obtención de productos farmacéuticos, mientras que los habitantes de los PD erogan generalmente más de 80 dólares al año en medicamentos (Lauridsen, 1988). La situación es aún más deplorable si consideramos que los PMD, en su mayoría con un control sanitario deficiente, emplean los magros recursos con que cuentan en la adquisición de medicamentos catalogados por comités de expertos en terapéutica como innecesarios, inútiles o francamente dañinos (Rivas \& Brito, 1984; DuránGonzález et al.,1990).

A diferencia de los PD, en donde el consumo de medicamentos es pagado casi totalmente por sistemas de seguros, en los PMD el gasto farmacéutico se financia entre el $50 \%$ y el $90 \%$ a través gasto familiar (WHO/DAP, 1997).

El consumo total de productos farmacéuticos en México es cercano a los US\$3,000 (tres millones de US dólares) en 1996 (IMS, 1996). El gasto en medicamentos representa el $45 \%$ del gasto total en salud; este consumo en relación al PIB es de 0,9\%, semejante al promedio latinoamericano, el cual es un tercio superior al de los PD (Murray \& Lopez, 1994).

En México, se ha observado que el empleo delos medicamentos no se realiza en condiciones adecuadas, esto es, mediante un diagnóstico correcto y con la prescripción del medicamento de primera elección (Rivas, 1992). Además, existen pocos estudios sobre los precios de los medicamentos, pero, en diferentes épocas, se ha encontrado que el precio de los medicamentos esenciales es elevado (De María \& Campos, 1981; Brudon, 1987; Molina, 1995).

Encontramos en México una disminución creciente de la capacidad adquisitiva de la población como resultado de la agudización de la crisis económica a partir de 1994 - el efecto “Tequila”. Los autores estiman, en un estudio, que la capacidad adquisitiva en medicamentos se redujo en 2 tercios, en promedio, para todos los tipos de medicamentos, en el periodo de junio 1993 a noviembre 1996 (Rivas et al., 1996).

¿Cuál es el precio razonable a pagar por un medicamento? es un asunto de una gran con- 
troversia en la sociedad. Las empresas mantienen precios elevados con base en el argumento de recuperar los costos de investigación y desarrollo, mientras que, en el caso de los pacientes-consumidores de los PMD, el problema radica en emplear adecuadamente parte de sus pequeños ingresos en la compra de medicamentos. Los organismos de consumidores y las agencias internacionales como la OMS propugnan por el acceso a los medicamentos para todas las personas, en especial de los medicamentos esenciales, como parte de la estrategia de salud para todos.

Por lo tanto, es importante estudiar el precio de los medicamentos en México, más aún cuando se estima que más del $85 \%$ de los medicamentos se comercializan en sector privado mediante el empleo de marcas comerciales y a precios de mercado (IMS, 1996).

El objetivo de este trabajo es estudiar el precio de los medicamentos esenciales de marca en México en relación al precio de los medicamentos esenciales genéricos disponibles en el mercado internacional, y además proporcionar información del contexto del mercado farmacéutico y socioeconómico mexicanos.

\section{Materiales y métodos}

Se estudió una muestra de 25 especialidades farmacéuticas de uso común que reúnen dos características: por un lado, estar incluidas en la Lista Modelo de Medicamentos Esenciales de la OMS (WHO, 1997) y, por otro lado, corresponder con la marca de mayor venta en sus categorías terapéuticas (IMS, 1996). Los precios nacionales se tomaron de acuerdo a la lista de precios de julio de 1995 de la proveedora Autrey (Autrey, 1995); esta lista registra los precios autorizados por el gobierno mexicano para cada uno de los medicamentos.

Los precios internacionales se tomaron de la International Dispensary Association (IDA), reportados para 1995 en The International Drug Price Indicator Guide (McFayden, 1995).

La comparación se hizo considerando los precios calculados para ventas al mayoreo de las unidades farmacéuticas de masa o volumen, esto es, tabletas o cápsulas para presentaciones farmacéuticas sólidas, o mililitros para presentaciones líquidas orales o parenterales; tanto para las especialidades farmacéuticas mexicanas como para los medicamentos de mercado internacional. En el caso de las especialidades farmacéuticas mexicanas, al costo en farmacia se le disminuyó un $28,7 \%$ como factor de ajuste, que corresponde a la diferen- cia de operación en las ventas al mayoreo y al menudeo (Molina, 1995). A los precios de los medicamentos en mercado internacional se les agregó un $20 \%$ como costo calculado promedio del seguro y colocación en el mercado mexicano.

\section{Resultados y discusión}

Los resultados son mostrados en la Tabla 1. Diez productos de marca $(40,0 \%)$ tuvieron un sobreprecio sobre el mercado internacional mayor al $500 \%$, en 4 productos $(20,0 \%)$ el sobreprecio fue mayor a $1000 \%$. Sólo en 7 productos (28,0\%) el sobreprecio fue menor a $200 \%$.

Los medicamentos estudiados se encuentran ya sin protección patentaria. Los precios de marca nacionales son un reflejo de un mer-

Tabla 1

Comparación de los precios de los medicamentos esenciales de marca en México con los precios de los genéricos en el mercado internacional, 1995.

\begin{tabular}{|c|c|}
\hline Medicamentos & $\begin{array}{l}\text { Precio de marca/ } \\
\text { Precio de Genéricos }\end{array}$ \\
\hline Amitriptilina (tabletas $25 \mathrm{mg}$ ) & 22,18 \\
\hline Hidroclorotiazida (tabletas 50 mg) & 20,82 \\
\hline Salbutamol (tabletas $4 \mathrm{mg}$ ) & 12,33 \\
\hline Paracetamol (acetaminofeno) (tabletas 500 mg) & 10,61 \\
\hline Propanolol (tabletas 40 mg) & 9,79 \\
\hline Acido acetil salicílico (tabletas 300 mg) & 9,40 \\
\hline Clorfeniramina (tabletas $4 \mathrm{mg}$ ) & 8,44 \\
\hline Gentacimina (Sol. inyectable $40 \mathrm{mg} / \mathrm{ml}$ ) & 7,14 \\
\hline Prednisona (tabletas $5 \mathrm{mg}$ ) & 6,87 \\
\hline Carbamacepina (tabletas $200 \mathrm{mg}$ ) & 5,38 \\
\hline Cloramfenicol (tabletas 250 mg) & 4,75 \\
\hline Sulfametoxazol + trimetoprima (tabletas $400 / 80 \mathrm{mg}$ ) & 4,52 \\
\hline Griseofulvina (tabletas $250 \mathrm{mg}$ ) & 4,19 \\
\hline Isosorbida dinitrato (tabletas $5 \mathrm{mg}$ ) & 3,42 \\
\hline Metronidazol (tabletas 250mg) & 3,17 \\
\hline Tetraciclina (unguento oftálmico 1\%) & 3,10 \\
\hline Fenitoína (tabletas 100 mg) & 2,89 \\
\hline Amoxicilina (tabletas $500 \mathrm{mg}$ ) & 2,19 \\
\hline Ampicilina (Polvo para inyección 500 mg) & 1,90 \\
\hline M etildopa (tabletas 250 mg) & 1,63 \\
\hline Acetazolamida (tabletas $250 \mathrm{mg}$ ) & 1,38 \\
\hline Eritromicina (tabletas $250 \mathrm{mg}$ ) & 1,15 \\
\hline Acido nalidíxico (tabletas 500 mg) & 1,06 \\
\hline Timolol (Sol. oftálmica 0,25\%) & 1,03 \\
\hline Gentamicina (Sol. oftálmica 0,3\%) & 0,75 \\
\hline
\end{tabular}

Fuente: Elaboraciones propias en base a precios de la International Dispensary Association (IDA), reportados para 1995 en The International Drug Price Indicator Guide, publicado por Management Sciences for Health (M cFayden, 1995). Los precios nacionales se tomaron de acuerdo a la lista de precios de julio de 1995 de la proveedora Autrey (Autrey, 1995). 
cado oligopólico, con una alta concentración por mercados terapéuticos según se ilustra en la Tabla 2.

Los medicamentos estudiados se encuentran sin protección patentaria, y los precios internacionales presentes en la lista que ofrece McFayden (1995) son una muestra de los precios de un mercado en competencia, en el cual las patentes ya no existen y los medicamentos se comercializan bajo su denominación genérica. Así esta lista se puede utilizar como un elemento de referencia para comparar los precios de los medicamentos nacionales con los precios en el mercado internacional.

La diferenciación de productos mediante marcas es fundamental en la estructura del mercado farmacéutico en México, donde la prescripción se lleva a cabo exclusivamente mediante el empleo de marcas y no de denominaciones genéricas. Recientemente, en México, se modificó la Ley General de Salud (Poder Ejecutivo Federal, 1997) por la cual se esta-

Tabla 2

Concentración en mercados terapéuticos seleccionados en México en 1996.

\begin{tabular}{lccc}
\hline $\begin{array}{l}\text { Categoría terapéutica } \\
\text { (n total de marcas) }\end{array}$ & $\begin{array}{r}\text { Porcentaje del total de ventas (n de marcas) } \\
(1)\end{array}$ & $(2)$ & $(3)$ \\
\hline Bloqueadores beta (13) & 30,2 & 51,9 & 69,0 \\
Vasodilatadores cerebrales (24) & 27,8 & 50,1 & 60,9 \\
Diuréticos (14) & 31,1 & 45,8 & 59,5 \\
Polivitaminas con minerales (52) & 22,3 & 40,5 & 54,0 \\
Hipocolesteromiantes (16) & 18,9 & 37,5 & 53,7 \\
Antiulcerosos (46) & 19,7 & 37,6 & 51,2 \\
Antidiabéticos orales (25) & 23,1 & 35,2 & 46,2
\end{tabular}

Fuente: Elaboraciones propias en base a cifras de IMS, 1996.

Tabla 3

Ingreso de la población económicamente activa en México en 1996.

\begin{tabular}{lr}
\hline U.S. dólares/día & $\%$ \\
\hline ninguno & 7,2 \\
2.9 o menos & 19,7 \\
$3.0-5.8$ & 36,2 \\
$5.9-8.7$ & 15,1 \\
$8.8-14.5$ & 9,7 \\
$14.6-29$ & 5,1 \\
más de 29 & 2,5 \\
desconocido & 4,5 \\
total & 100,0 \\
\hline
\end{tabular}

Fuente: Elaboraciones propias, con base en la información publicada por el Instituto Nacional de Geografía y Estadística (INEGI), 1996. blece la obligatoriedad del uso de las denominaciones genéricas, tanto en la prescripción como en el envase de los productos.

La agudización de la crisis económica mexicana en 1994 provocó una caída del PIB, en 1995, a -6\%; lo que determinó, junto con una caída muy importante de la actividad económica de Argentina, un crecimiento promedio latinoamericano de $0,4 \%$ en el mismo año (CEPAL, 1996). Sin embargo, este descenso del PIB no se reflejó proporcionalmente en el gasto en medicamentos per cápita, el cual se ha mantenido, en los últimos 5 años, de acuerdo a cálculos de los autores, entre 25 y 30 dólares per cápita.

Dado que más del $60 \%$ de la población económicamente activa tiene un ingreso diario inferior a 6 dólares (Tabla 3) (INEGI, 1996), se estima que estos sectores de población de más bajos ingresos tienen un consumo farmacéutico muy reducido o nulo. En conversación con uno de los autores, un directivo de una multinacional comentó que “la ganancia es igual al producto del precio del medicamento por las unidades vendidas; $y$, si por la reducción del ingreso disminuye el número de unidades vendidas, entonces hay que elevar el precio para mantener los niveles de ganancia". Esto define la estrategia de la industria farmacéutica de mantener sus tasas de ganancia a expensas de las ventas a los sectores de población de mayores ingresos, con la consiguiente disminución del poder de compra de los sectores poblacionales de menores ingresos.

Asimismo, la planta productora de medicamentos de países como Argentina, Brasil y México tiene como características comunes principales las siguientes: i) la operación con una gran cantidad de materias primas importadas, con altos precios de transferencia y con elevados pagos de regalías al exterior; ii) esfuerzos nacionales pequeños en investigación y desarrollo y iii) diferencias en el tipo de especialidades farmacéuticas ofrecidas en los países latinoamericanos y en los países donde se encuentran las casas matrices (CEPAL, 1987).

Los resultados mostrados no requieren explicaciones adicionales y muestran que, a pesar de la severa crisis económica mexicana, los márgenes de ganancia de las empresas farmacéuticas se mantienen muy el evados. La conveniencia de establecer un mercado genérico en los países latinoamericanos ha sido discutida recientemente (Bermudez, 1994). En países donde coexisten en el mercado medicamentos de marca y medicamentos genéricos, se observan diferencias de precios importantes entre unos y otros, permitiendo al consumidor tener 
opciones. En un estudio no publicado, los autores encontraron que los precios por unidad farmacéutica de medicamentos de marca y genéricos, en los Estados Unidos de América, de acuerdo a un catálogo de precios de ese país (Red Book, 1996), difieren en forma muy importante; en algunos casos, el medicamento de marca tiene un precio superior al genérico hasta en más de $2000 \%$. Algunos ejemplos notables de estos sobreprecios son el metronidazol (2290\%), la clorfeniramina (2100\%), la hidroclorotiazida (1330\%) y el diacepan (960\%).

\section{Conclusiones y propuestas}

La reducción de los precios de los medicamentos, para asegurar un acceso equitativo, es una necesidad urgente en M éxico, dado el bajo ingreso per cápita y el bajo porcentaje de población asegurada. Una competencia de "mercado perfecto" permitiría una equilibrio apropiado entre los precios pagados por los consumidores y la producción. Pero las condiciones que permiten este "mercado perfecto" no se dan en México. El mercado farmacéutico mexicano es altamente concentrado, hay una diferenciación muy acentuada de productos por la prescripción basada exclusivamente en marcas, sin la existencia de un mercado genérico; esto se debe, entre otros factores, a una dificultad importante para la entrada de firmas farmacéuticas fabricantes de genéricos.

Si bien la comparación de precios entre productos de marca y productos disponibles en mercado internacional es cuestionada por la industria farmacéutica transnacional, el análisis hecho lo consideramos válido para el ámbito de medicamentos que ya han tenido un periodo de explotación exclusiva, mediante el uso de patentes y marcas, por los laboratorios que los desarrollaron. $Y$, de esta manera, estos laboratorios han recuperado los costos de investigación y desarrollo y posteriormente podrán seguir teniendo un porcentaje importante en un mercado de competencia en genéricos.

A la estrategia de los genéricos que comienza a implantar el gobierno mexicano deberán seguir una serie de medidas que permitan la creación de hábitos de prescripción genérica, con campañas educativas dirigidas a médicos y consumidores.

Las reformas iniciadas deberán también ser completadas con medidas que favorezcan un abastecimiento adecuado de medicamentos genéricos de calidad apropiada. Estos medicamentos genéricos podrán ser fabricados en el país o comprados en el mercado internacional a proveedores certificados por organismos internacionales como la OMS o Unicef.

\section{Referencias}

AUTREY, 1995. Lista de Precios de Especialidades Farmacéuticas (julio, 1995). México: Autrey.

BERMUDEZ, J., 1994. Medicamentos genéricos: uma alternativa para o mercado brasileiro. Cadernos deSaúdePublica, 10:368-378.

BRUDON, P., 1987. ¿Medicamentos para Todos en el Año 2000?. México: Siglo XXI.

BURSTALL, M. L., 1990. The Regulation of the Pharmaceutical Industry. London: IEA Health Series no 9.

CEPAL (Comisión Económica para América Latina y el Caribe), 1987. La Industria Farmacéutica y Farmacoquímica: Desarrollo Histórico y Posibilidades Futuras. Santiago de Chile: CEPAL, Naciones Unidas.

CEPAL, 1996. Panorama Económico de América Latina 1996. Santiago de Chile: CEPAL.

CHETLEY, A., 1989. A Healthy Business?. London: ZED.

DE MARIA Y CAM POS, M., 1981. La Industria Farmacéutica en México. Serie de Lecturas del FCE no 34. México: FCE. 
DURAN-GONZALEZ, L.; BECERRA, J.; FRANCO, F.; KRAVZOV-JINICH, J.; VISO-GUROVICH, F. \& FRENK-MORA, J., 1990. Uso cuadro básico de medicamentos en el primer nivel de atención, Salud Pública de México, 32:543-551.

IMS (International Marketing Service), 1996. The Pharmaceutical Market in Mexico. Zug: IMS.

INEGI (Instituto Nacional de Geografía y Estadística), 1996. Estadísticas sobre Empleo en México 1996. México: INEGI.

LAURIDSEN, E., 1988. Medicamentos esenciales: concepto y estrategias de implantación global. I Conferencia Latinoamericana sobre Políticas Farmacéuticas y Medicamentos Esenciales, resúmenes. México.

MOLINA, R., 1995. Estructura de Mercado y Precios. El caso de los Medicamentos Esenciales en la Industria y las Finanzas en el México Actual. M éxico: Departamento de Economía de la UAM (Universidad Autónoma Metropolitana).

MCFAYDEN, J. E., 1995. The International Drug Price Indicator Guide. Arlington: Management Sciences of Health.

MURRAY, C. J. L. \& LOPEZ, A. D., 1994. Global Comparative Assessments in the Health Sector. Disease Burden, Expenditures, and Intervention Packages. Geneva: World Health Organization.

PATEL, M., 1983. Drug costs in developing countries and policies to reduce them. In: Pharmaceuticals $\&$ Health in the Third World (S. J. Patel, ed.), pp. 36-45, Oxford: Pergamon.

PODER EJECUTIVO FEDERAL, 1997. Decreto por el que se reforma la Ley General de Salud. Diario Oficial de los Estados Unidos Mexicanos, 7 mayo: 5-9. México: Poder Ejecutivo Federal.
RED BOOK, 1996. Drug Topics. Pharmacy's Fundamental Reference. Oradell: Medical Economics.

RIVAS, J. \& BRITO, A., 1984. Metodología para el estudio de los patrones de prescripción demedicamentos. Reporte de Investigación 40. México: Universidad Autónoma Metropolitana-Xochimilco.

RIVAS, J., 1992. Patrones de prescripción y educación médica. En: Medicamentos, Economía y Salud ( $R$. Molina \&J. D. F. Rivas, orgs.), pp. 169-179, México: Universidad Autónoma Metropolitana-Iztapalapa.

RIVAS, J.; M OLINA, R. \& GARZA, P., 1996. Aspectos de la utilización de medicamentos en México. Análisis del periodo 1988-1996. In: Temas Sel ectos de Investigación Clínica II, pp. 133-144, México: Universidad Autónoma Metropolitana-Xochimilco.

WHO (WORLD HEALTH ORGANIZATION), 1975. Resolutions and decisions. World Health Assembly. Geneva: WHO.

WHO (WORLD HEALTH ORGANIZATION), 1977. The Selection of Essential Drugs. Report of a WHO Expert Committee. Technical Report Series, No. 615. Geneva: WHO.

WHO (WORLD HEALTH ORGANIZATION), 1981. Global strategy for health for all by the year 2000. 34th World Health Assembly. Geneva: WHO.

WHO (WORLD HEALTH ORGANIZATION), 1997. The Use of Essential Drugs: Model List of Essential Drugs (Ninth List). Seven Report of WHO Expert Committee. Technical Report Series, no 867. Geneva: WHO.

WHO (WORLD HEALTH ORGANIZATION), 1997. Global Comparative Pharmaceutical Expenditures. Health Economics and Drugs. WHO/DAP Series no 3. Geneva: WHO. 